\begin{tabular}{|c|l|}
\hline Title & Action spectrum analyses of photoinduced superhydrophilicity of titania thin films on glass plates \\
\hline Author(s) & Y an, X.; A be, R.; Ohno, T.; Toy ofuku, M.; Ohtani, B. \\
\hline Citation & $\begin{array}{l}\text { Thin Solid Films, 516(17), 5872-5876 } \\
\text { https://doi.org/10.1016/.ts.2007.10.033 }\end{array}$ \\
\hline Issue Date & 2008-07-01 \\
\hline Doc URL & http://hdl.handle.net/2115/44875 \\
\hline Type & article (author version) \\
\hline File Information & TSF516-17_5872-5876.pdf \\
\hline
\end{tabular}

Instructions for use 


\title{
Action spectrum analyses of photoinduced superhydrophilicity of titania thin films on glass plates
}

\author{
X. Yan ${ }^{\mathrm{a}}$, R. Abe ${ }^{\mathrm{a}}, \mathrm{T} . \mathrm{Ohno}^{\mathrm{b}}$, M. Toyofuku ${ }^{\mathrm{b}}$, B. Ohtani ${ }^{\mathrm{a} *}$ \\ ${ }^{a}$ Catalysis Research Center, Hokkaido University, Sapporo 001-0021, Japan \\ ${ }^{\mathrm{b}}$ Department of Material Science, Faculty of Engineering, Kyushu Institute of Technology, 1-1 \\ Sensuicho, Tobata, Kitakyushu 804-8550, Japan
}

*Corresponding author: Tel.: +81-11-706-9132, Fax: +81-11-706-9133. E-mail address: ohtani@cat.hokudai.ac.jp

\begin{abstract}
Samples of titania-thin-film-coated glass plates were prepared by spin coating of commercial titania-coating liquids using a home-made spin coater (1000 rpm, $30 \mathrm{~s})$ and then calcined in air at $673 \mathrm{~K}$ for $2 \mathrm{~h}$. In a part of experiments, samples were stored in an oven at $393 \mathrm{~K}$ overnight. A piece of sample was set in a $10-\mathrm{mm}$ rectangular quartz cell, and monochromatic light (FWHM of ca. $17 \mathrm{~nm}$, intensity of ca. $10^{8}$ einstein $\mathrm{s}^{-1}$ ) was irradiated in ambient air using a grating multiple-wavelength photoirradiation instrument (Jasco CRM-FD). Water contact angle (WCA) of samples before and after monochromatic irradiation was measured by a contact angle analyzer. Changes in WCA, as a function of wavelength of monochromatic irradiation, a kind of action spectrum, of several titania samples showed that the sample surfaces became superhydrophilic, i.e., WCA became almost zero, only under irradiation with a wavelength shorter than the absorption edge, corresponding to the band gap, of samples. Analysis of changes in WCA showed that there are at least two stages when the samples stored at $393 \mathrm{~K}$ overnight before irradiation to give relatively high WCA of ca. 40 deg. were used: first stage of WCA $>10$ deg. and second stage of WCA $<10$ deg.. The
\end{abstract}


action spectra of WCA change in these two stages are discussed.

Keywords: Titania thin film; Spin coating; Photoinduced superhydrophilicity, Water contact angle, Photocatalytic reaction,

\section{Introduction}

Photocatalytic reaction is a chemical reaction induced by photoexcited electrons and positive holes generated in photoirradiated semiconducting (or sometimes insulating) materials [1] and has been applied practically, e.g., for decomposition of organic and inorganic contaminants in air and water [2]. Coating of window-glass plates and outdoor walls with a photocatalyst is effective for keeping the surface clean. Such an effect is called "self-cleaning". It has been proposed that two phenomena are involved in self-cleaning: photocatalytic complete oxidative decomposition of chemicals, i.e., "mineralization" on the surface, and photoinduced hydrophilicity of the surface, i.e., water contact angle (WCA) becoming almost zero due to photoirradiation, to make the surface easily washable by rain [3]. The mechanism of the former phenomenon, photocatalytic oxidative decomposition, has been almost clarified and that of the latter, photoinduced superhydrophilicity, has been partly clarified, i.e., changes in the structure of the photocatalyst surface due to photogenerated positive holes [2].

Titania, titanium(IV) oxide, has been widely used as a stable, robust and inexpensive photocatalyst, which absorbs light of wavelength < ca. $400 \mathrm{~nm}$, i.e., ultraviolet light [5]. For the photocatalytic mineralization as well as chemical reactions other than superhydrophilicity, it has been proved that the reactions are initiated through the above-mentioned band-gap excitation followed by redox reactions with electron-hole pairs through action spectrum analyses, i.e., studies on wavelength dependence of photochemical or photocatalytic reaction rates $[3,4]$. On the other hand, as far as we know, there have been no such action spectrum analyses of photoinduced superhydrophilicity. In order to confirm that redox reactions by photogenerated positive holes or electrons initiate the photoinduced superhydrophilicity, 
action spectra of photoinduced changes in WCA were measured for titania-coated glass substrates in the present study.

\section{Experimental}

\subsection{Pretreatment of glass slide substrate}

Glass plates (Matsunami, S-1111 micro slide glass) were used as support substrates and were pretreated before coating. First, they were rubbed with silica $\left(\mathrm{SiO}_{2}\right)$ powder (99.99\%, Wako Pure Chemical Industries) to enhance their surface roughness, followed by rinsing with deionized $\mathrm{RO}$ water. Without this rubbing process, homogeneous coating of titania could not be obtained. Then the plates were ultrasonically cleaned for $10 \mathrm{~min}$ in acetone. Finally, they were kept in acetone and dried with argon blowing before coating.

\subsection{Film preparation}

In the present work, five kinds of titania sols were used for film preparation. M-6 (anatase, $30 \mathrm{wt} \%$, average particle size of $7 \mathrm{~nm}$, Taki Chemical Co. Ltd.), PC-201 (anatase, 20 wt\%, average particle size of 20 nm, Titan Kogyo, Ltd.), NTB-01 (brookite, 10 wt\%, particle size of 10-35 nm, Showa Titanium), and NTB-03 (brookite, $6 \mathrm{wt} \%$, particle size of 10-35 nm, Showa Titanium) were gifts from suppliers, and sulfur-doped titania $\left(\mathrm{S}-\mathrm{TiO}_{2}\right)$ and its sol solution were preparing according to the literature [5]. Titania thin films were prepared by spin-coating using a home-made spin coater. First, a $0.10-\mathrm{mL}$ portion of titania sol was poured on a glass plate and then spinning at $1000 \mathrm{rpm}$ was performed for $30 \mathrm{~s}$. The coated glass plate was dried at $393 \mathrm{~K}$ for $10 \mathrm{~min}$ and then cooled to room temperature. The coating-drying cycles were repeated to obtain desired thickness. Finally, the films were annealed in an electric furnace (Shirota, P-2S) at $673 \mathrm{~K}$ for $2 \mathrm{~h}$. These titania-coated films were almost transparent. In the part of experiments, samples were stored in a vacuum drying oven (Fine, FVO-10) at $393 \mathrm{~K}$ under an argon atmosphere (with slightly reduced pressure) for at least $12 \mathrm{~h}$ before use.

\subsection{Monochromatic irradiation}

The glass plates coated with titania thin films were cut into several pieces $(9 \mathrm{~mm}$ in width and $26 \mathrm{~mm}$ in height) just before irradiation. A sample piece was placed in a rectangular 
quartz cell (10 mm square and $50 \mathrm{~mm}$ in height) and irradiated at monochromatic wavelengths using a diffraction grating-type illuminator (Jasco, CRM-FD) equipped with a 300-W xenon lamp (Hamamatsu, C2578-02). Several stainless-steel screens with different mesh sizes were placed before the quartz cells to adjust monochromatic light intensity to almost the same for the overall wavelength range. The light intensity was measured by an optical power meter (HIOKI 3664). In the preliminary experiments, it was found that WCA of a part of the sample surface once wetted gives different WCA after photoirradiation when compared with that of another part not wetted. Therefore, we took care not to use the once-wetted part for WCA measurement.

\subsection{Photoabsorption spectrum measurement}

Absorption spectra of samples were recorded using a Shimadzu MPS-2450 multi-purpose spectrophotometer with spectral resolution of $2 \mathrm{~nm}$. The measurements were performed in transmission mode and recorded as \%transmission, $100 I / I_{0}$, where $I$ and $I_{0}$ represent relative intensity of transmitted light through the glass plates with and without a titania-coating layer.

\subsection{Water contact angle measurement}

The surface hydrophilicity-hydrophobicity was evaluated by measuring WCA using a contact angle analyzer (Kyowa Interface Science, DropMaster 500) under ambient conditions. A sessile drop method was used for contact angle measurements, and the volume of a water droplet in each measurement was $0.001 \mathrm{~mL}$.

\section{Results and discussion}

\subsection{Absorption spectra of titania-coated glass plates}

Figure 1 shows absorption spectra of 4 samples in ultraviolet and visible-light ranges. As expected, light of wavelength < ca. 370-400 nm, corresponding to the band gap of titania, was absorbed. Shift of absorption onset was observed depending on the kind of commercial titania coating liquids; the onset wavelength was red-shifted in the order of M-6, NTB-3, NTB-1 and PC-201. In the preparation of coated plates, the number of repeated spin-coating processes was fixed to 5 for all of the samples. Since the contents of titania in the coating 
liquid, as well as viscosities of liquid, were different, the thicknesses of titania thin films might be different. Neglecting this and assuming that anatase (M-6 and PC-201) and brookite (NTB-1 and NTB-3) crystallites have almost the same band-gap energy in bulk crystal $[6,7]$, the onset wavelength seemed to become shorter with decrease in particle size (see Experimental).

3.2 Change in WCA for titania-coated glass plates without low-temperature storage under monochromatic irradiation.

For all of the samples prepared using commercial titania sol solutions used in this study, the initial WCA before the monochromatic irradiation was ca. 10 deg., relatively low compared with that of single crystal titania [2]. WCA depends on not only the chemical composition of the solid surface, e.g., density of surface hydroxyls, but also macroscopic structure, e.g., surface roughness; it is well known that porosity of solids enhances both hydrophilicity and hydrophobicity. Although at present we have no structural information on the samples prepared and used in this study, such sol-gel derived samples must be sufficiently porous to enhance their original hydrophilicity. Sakai et al. reported that ultrasonication increased WCA of a titania thin film prepared by spin coating [8]. However, in our studies, no such effect was observed. The reason for this difference has not been clarified yet. In the first part of this study, thin films with WCA of ca. 10 deg. were irradiated with monochromatic light.

Monochromatic irradiation induced a change in WCA, the degree of which depended strongly on the wavelength of irradiation. The results by 2-h irradiation are shown in Figure 2. This may be called as "action spectra" for photoinduced hydrophilicity. To the best of our knowledge, this is the first example showing wavelength dependence for photoinduced hydrophilicity, though there has been a report showing hydrophilic/hydrophobic behavior of a titania surface depending on the irradiation of ultraviolet light and visible light [2]. However, in a strict sense, an action spectrum should be a plot of apparent quantum efficiency as a function of wavelength or photon energy of irradiation. Apparent quantum efficiency is defined as the ratio of molar amount of chemical change, formation of product or consumption of substrate(s), and molar amount (exactly speaking, measured in units of 
"einstein") of incident photons. The mechanism of photoinduced change in surface hydrophilicity has been investigated, and it has been shown that the superhydrophilicity is induced by an increase in number of surface hydroxyls due to the reaction of positive holes and thereby the phenomenon may be caused by chemical reaction(s). However, WCA does not correspond directly to the molar amount of such chemical species. Since the molar ratio of chemical species and incident photons is calculated in conventional action-spectrum measurements, the use of monochromatic light beams of different intensities is allowed. On the other hand, we cannot compensate the light-intensity difference by dividing WCA with the intensity. Therefore, the light intensity had to be adjusted, rather strictly, to be the same in the measurements. For this adjustment, there are alternative ways, adjustment in the energy, e.g., measured in units of $\mathrm{W}$ and in the number of photons, e.g., in units of einstein $\mathrm{s}^{-1}$. In this study, the latter, photon flux, was used for adjustment. The actual light intensity of monochromatic light was $1.54-2.26 \cdot 10^{8}$ einstein $\mathrm{s}^{-1}$ (ca. $7-4 \mathrm{~mW} \mathrm{~cm}^{-2}$ ) after adjustment by reducing original light intensity using stainless-steel meshes.

Thus, in a strict sense, it is not so easy to measure an action spectrum for photoinduced superhydrophilicity, but the present results are consistent with the proposed mechanism, i.e., photogenerated electron-positive hole pairs induce the superhydrophilicity.

3.3 Change in WCA for titania-coated glass plates stored in an oven of relatively low temperature

During this study, we found that storage of titania-coated plates in an oven at $393 \mathrm{~K}$ overnight increases WCA to more than $30 \mathrm{deg}$. Difference in atmospheres of heating gave almost no effect on WCA after heating, i.e., before the monochromatic irradiation; heating in ambient air, dried air, argon, or under vacuum induced an increase in WCA. Since the samples must be handled in ambient air during monochromatic irradiation and WCA measurement, surface contamination could not be avoided, i.e., samples having clean surfaces cannot be obtained. At present we have no analytical evidence, the WCA increase might be induced by contamination of the sample surface during the heating with organic compounds in the oven. This will be discussed later.

Figure 3 shows the time courses of changes in WCA of samples ( $\mathrm{M}-6$ and $\left.\mathrm{S}-\mathrm{TiO}_{2}\right)$ by 
monochromatic irradiation at five different wavelengths from 320 to $380 \mathrm{~nm}$. For both samples, each time-course curve had an inflecting point at around $10 \mathrm{deg}$. of WCA; in the WCA range > ca.10 deg. (Stage 1), the rate of WCA change depended on the wavelength of monochromatic light, while in the WCA range < ca. 10 deg. (Stage 2), the rate seemed to be independent of the wavelength. Except for WCA of the boundary of 10 deg., the WCA change was almost linear with respect to irradiation time. It has been reported that the reciprocal of WCA increased linearly with time of photoirradiation [9] on titania films prepared by dip-coating of sol solutions. The differences in titania films used in the present study and those used in that study was the absence and presence of silica underlayers, but this might not account for the difference in time courses. In this study, the rate of changes in WCA in each stage for each sample was evaluated by fitting the time-course curve to a linear line and is shown using units of deg. $\min ^{-1}$.

Figure 4 shows the rates of WCA change as a function of irradiation wavelength in both stages. It is clearly shown that the rates in Stage 1 for both titania-coatings depended strongly on the irradiation wavelength, while those in Stage 2 seemed almost independent of the wavelength. For the rates in Stage 1 for M-6 and S- $\mathrm{TiO}_{2}$ samples, these action spectra resembled those of absorption spectra and the onset wavelength for $\mathrm{S}-\mathrm{TiO}_{2}$ was shifted to a longer wavelength when compared with that for M-6. These results suggest that the decrease in WCA in Stage 1 is attributable to photocatalytic reaction occurring on the photoirradiated titania surface. As was described in the preceding section, these samples had been stored in a drying oven to increase the initial WCA, and the WCA increase might be caused by surface contamination with organics. It seems reasonable that such contaminants were decomposed photocatalytically to expose the bare titania surface, which is essentially hydrophilic and, thereby, the action spectrum of WCA change resembles that of photocatalytic oxidative decomposition of organic compounds, such as acetic acid or methylene blue [10]. We tried to detect the change in the amount of surface contaminants by infrared or electronic absorption spectroscopy, but no reproducible results were obtained.

Although a suspension of $\mathrm{S}-\mathrm{TiO}_{2}$ powder has shown photocatalytic activity for acetic acid decomposition under visible light irradiation [10], no appreciable change in WCA was 
observed in this study under visible-light irradiation at wavelengths $>400 \mathrm{~nm}$. Since it has been reported that similar doped-titania coatings showed a rate of WCA change approximately two orders of magnitude lower than that under ultraviolet irradiation $[11,12,13]$, we cannot conclude that $\mathrm{S}-\mathrm{TiO}_{2}$ has no visible-light response for photoinduced superhydrophilicity.

Opposite to the above-mentioned behavior in Stage 1, the rates of WCA change in Stage 2 seemed independent of the irradiation wavelength for both samples. At present we have no information on the change of chemical composition in Stage 2 and we can only suggest that the photoinduced change in WCA in Stage 2 is rather complicated and cannot be explained by the photocatalytic decomposition of surface contaminants as was the case in Stage 1. As reported previously [2], visible-light irradiation increased WCA of titania thin films prepared by an MOCVD method that had undergone ultraviolet irradiation to become superhydrophilic. For the $\mathrm{S}-\mathrm{TiO}_{2}$ sample, the rate of WCA change under irradiation at 380 $\mathrm{nm}$ (Stage 1) was relatively small, but the rate in Stage 2 was comparable to that at $320 \mathrm{~nm}$ (Fig. 4). When this film was irradiated first at $320 \mathrm{~nm}$ to give WCA of ca. $10 \mathrm{deg}$. and then irradiated at $380 \mathrm{~nm}$, WCA was slowly increased, but not decreased as was observed in the case of continuous 380-nm irradiation. This indicates that the surface composition or structure of the film determining WCA is different depending on the irradiation wavelength in Stage 1 even though the sample shows similar WCA of 10 deg., and the mechanism of change in Stage 2 may be different depending on the surface composition or structure.

\section{Conclusions}

Wavelength dependence of photoinduced change in WCA of titania thin films was analyzed. Since WCA change could not be fully explained by change in chemical composition or structure of film surfaces, an action spectrum in a strict sense could not be obtained. However, it has been suggested that the superhydrophilicity is essentially induced by band-gap photoexcitation of as-prepared hydrophilic (with ca. 10 deg WCA) titania films. When the samples were stored in an oven at $393 \mathrm{~K}$ to become less hydrophilic (with ca. 40-50 deg. WCA) and then irradiated with monochromatic light, the initial WCA decrease (Stage 1) 
was induced presumably via photocatalytic decomposition of surface contaminants and dependent on the irradiation wavelength, while the WCA change below 10 deg. of WCA was independent of the wavelength and the mechanism could not be explained only by chemical reactions such as photocatalytic oxidative decomposition. Detailed and precise analyses of chemical composition and structure using, for example, double-beam photoacoustic spectroscopy (PAS) [14,15] before and during the course of photoirradiation are required for a full understanding of photoinduced hydrophilicity.

\section{References}

[1] A. Fujishima, Tata N. Rao and D. A. Tryk, J. Photochem. Photobiol. C: Photochem. Rev., 1 (2000) 1.

[2] M. Miyauchi, N. Kieda, S. Hishita, T. Mitsuhashi, A. Nakajima, T. Watanabe, K. Hashimoto, Surf. Sci., 511 (2002) 401 and references therein.

[3] T. Torimoto, N. Nakamura, S. Ikeda, B. Ohtani, Phys. Chem. Chem. Phys., 4 (2002) 5910.

[4] T. Torimoto, Y. Aburakawa, Y. Kawahara, S. Ikeda, B. Ohtani, Chem. Phys. Lett., 392 (2004) 220-224.

[5] T. Ohno, M. Akiyoshi, T. Umebayashi, K. Asai, T. Mitsui, M. Matsumura, Appl. Catal. A: Gen., 265 (2004) 115.

[6] B. Ohtani, J-i. Handa, S.-i. Nishimoto, T. Kagiya, Chem. Phys. Lett., 120 (1985) 292

[7] H. Kominami, Y. Ishii, M. Kohno, S. Konishi, Y. Kera, B. Ohtani, Catal. Lett., 91 (2003) 41.

[8] N. Sakai, R. Wang, A. Fujishima, T. Watanabe, K. Hashimoto, Langmuir, 14 (1998) 5918.

[9] N. Sakai, A. Fujishima, T. Watanabe, K. Hashimoto, J. Phys. Chem. B, 107 (2003) 1028.

[10] X. Yan, T. Ohno, K. Nishijima, R. Abe, B. Ohtani, Chem. Phys. Lett., 429 (2006) 606-610.

[11] H. Irie, S. Washizuka, N. Yoshino, K. Hashimoto, Chem. Commun. (2003) 1298. 
[12] H. Irie, S. Washizuka, K. Hashimoto, Thin Solid Films, 510 (2006) 21.

[13] H. Irie, S. Washizuka, Y. Watanabe, T. Kako, K. Hashimoto, J. Electrochem. Soc., 152 (2005) E351.

[14] N. Murakami, O. O. Prieto Mahaney, T. Torimoto, B. Ohtani, Chem. Phys. Lett., 426 (2006) 204.

[15] N. Murakami, O. O. Priet Mahaney, R. Abe, T. Torimoto, B. Ohtani, J. Phys. Chem. C., in press. 


\section{Figure captions}

Fig. 1. Photoabsorption spectra of titania thin-film coated glass plates in ultraviolet and visible regions measured in units of \%transmittance.

Fig. 2. Wavelength dependence of WCA after irradiation with monochromatic light on the titania thin-film coated glass plates.

Fig. 3. Time courses of changes in WCA of samples (upper: M-6 and bottom: $\mathrm{S}-\mathrm{TiO}_{2}$ ) by monochromatic irradiation at five different wavelengths from 320 to $380 \mathrm{~nm}$.

Fig. 4. Rates of WCA changes of samples (upper: M-6 and bottom: S- $\mathrm{TiO}_{2}$ ) as a function of irradiation wavelength. Black and grey bars represent the rates in Stage 1 and 2, respectively. 




Fig. 1. 


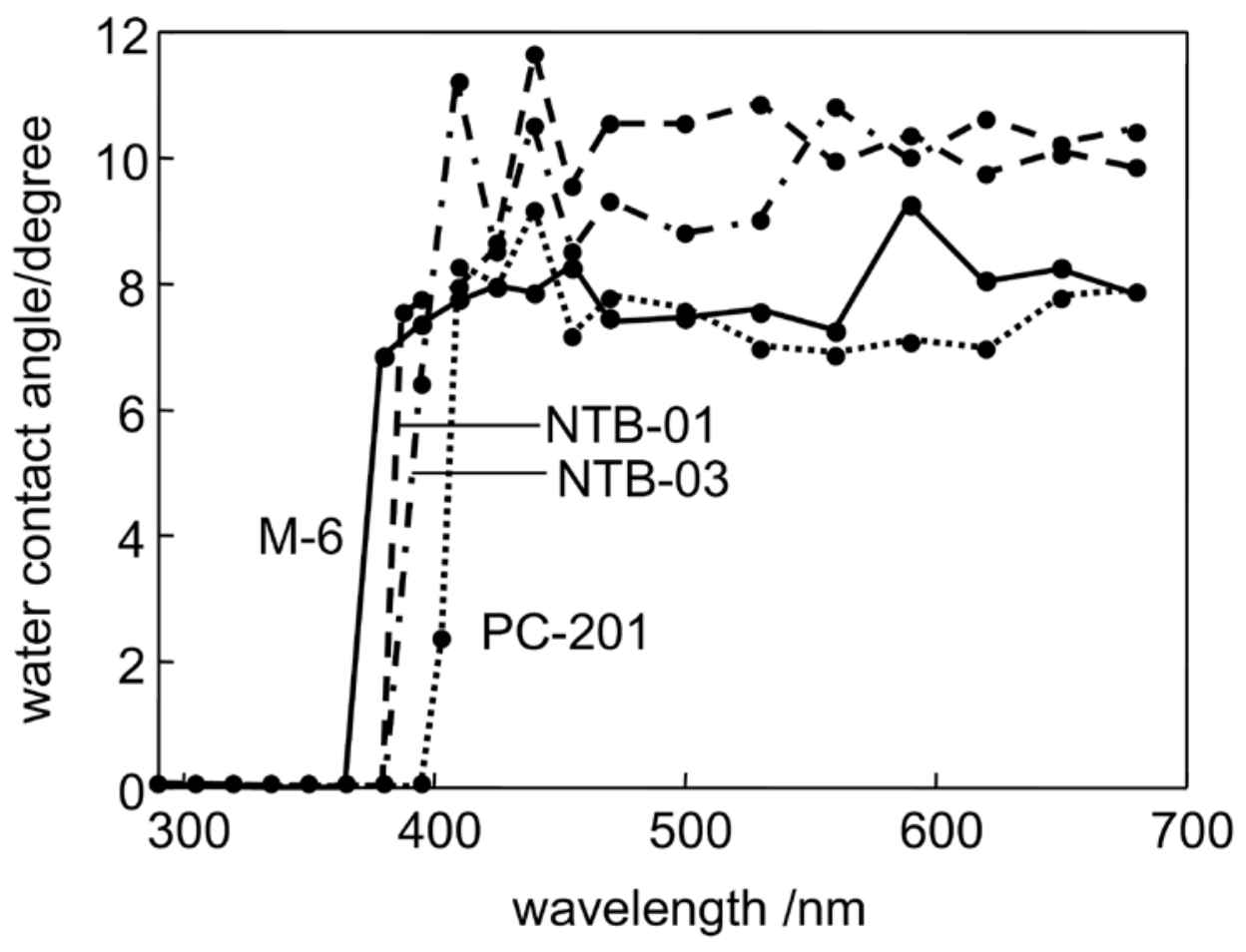

Fig. 2. 



Fig. 3. 



Fig. 4. 